Reprod. Nutr. Dévelop., 1984, 24 (3), 327-341.

\title{
Influence de la température ambiante sur l'utilisation métabolique de l'énergie et de l'azote alimentaires chez le rat en croissance
}

\author{
R. CHRISTON, J. LE DIVIDICH $\left({ }^{*}\right)$, B. SEVE $(*)$, A. AUMAITRE $(*)$
}

avec la collaboration technique de J. PEINIAU, D. DENOUE et C. BLONDEL

Station de Recherches zootechniques. I.N.R.A., C.R.A.A.G., 97170 Petit-Bourg, France.

(*) Station de Recherches sur /'Elevage

des Porcs, I.N.R.A.,

35590 Saint-Gilles (France).

Summary. Effect of ambient temperature on the metabolic use of dietary energy and nitrogen in growing rat.

Sixty-seven male rats of the Wistar CF strain were used in two trials to study the effect of ambient temperature on nitrogen and energy balances (experiment 1) and on the variations of certain biochemical parameters of metabolism (experiment 2). In both cases, the rats, housed individually in metal cages and fed ad libitum, were kept under three different temperatures : $21^{\circ} \mathrm{C}$ (control), $3{ }^{\circ} \mathrm{C}$ (cold), $31^{\circ} \mathrm{C}$ (hot).

Compared to the controls, the rats at $3{ }^{\circ} \mathrm{C}$ showed a significant increase $(P<0.01)$ in food intake and a significant decrease $(P<0.01)$ in daily growth rate and feed efficiency ratio ; body fat content was unchanged. Compared to the controls, the rats at $31^{\circ} \mathrm{C}$ showed a decrease $(P<0.01)$ in food intake and growth rate, a better feed efficiency ratio $(P<0.01)$ and a significant increase in carcass lipid content.

When compared to rats at $21^{\circ} \mathrm{C}$, those at $3{ }^{\circ} \mathrm{C}$ had a much lower nitrogen retention ; at $31{ }^{\circ} \mathrm{C}$ this retention was only slightly lower. The urinary nitrogen excretion, increased by the cold temperature, reflected higher nitrogen catabolism, while its increase at a high temperature was probably due to a decrease in protein synthesis, and a subsequent degradation of excess ingested nitrogen. Energy retention increased $(P<0.01)$ as the ambient temperature increased from 3 to $31{ }^{\circ} \mathrm{C}$, and wide variations in the amounts of fixed energy in the form of proteins or lipids were observed.

The balance results obtained have been discussed in relation to the variations of some biochemical parameters of metabolism such as free fatty acids, free glycerol, lipoproteinlipase activity and in vitro utilization of labelled glucose.

\section{Introduction.}

L'environnement thermique des animaux conditionne en grande partie leur croissance par son influence déterminante sur les échanges d'énergie entre la masse corporelle et le milieu ambiant. Au cours des vingt dernières années, ce sujet a donné lieu à de nombreux travaux et on sait maintenant que le gain de 
poids maximum est réalisé dans une gamme de températures qui dépend principalement de l'espèce, du poids corporel et de l'alimentation. Ainsi, ce maximum est observé entre 15 et $20^{\circ} \mathrm{C}$ pour le porc en croissance-finition (Jensen et al., 1963 ; Holmes et Close, 1977 ), entre 10 et $20^{\circ} \mathrm{C}$ pour le veau préruminant de 100 à 150 $\mathrm{kg}$ (Webster et al., 1976), aux environs de $12-13^{\circ} \mathrm{C}$ pour le mouton au pâturage (Webster, 1976), entre 19 et $25^{\circ} \mathrm{C}$ pour le rat blanc de laboratoire (Hiller et al., 1977). On doit toutefois remarquer que ces mesures peuvent être assez variables selon les conditions expérimentales et notamment la durée d'adaptation des animaux avant la période d'enregistrement ou, particulièrement lors d'expérimentations en plein air, l'incidence des autres facteurs climatiques tels que la pluie et le vent sur la température ambiante effective. II est également bien établi que, chez l'animal placé en dehors de sa zone de neutralité thermique, les pertes de chaleur liées à la thermorégulation affectent l'énergie fixée dans l'organisme, soit globalement, soit dans sa répartition en énergie retenue sous forme de protéines ou de lipides (Verstegen et al., 1973). La diminution de la quantité d'énergie disponible pour les synthèses s'accompagne alors, selon l'état nutritionnel, d'une réduction du gain de poids et, souvent, d'une modification de la composition corporelle (Verstegen et al., 1978).

Toutefois, dans la plupart des études réalisées sur les animaux de ferme, on s'est peu préoccupé des aspects physiologiques ou biochimiques des transformations induites par la température ambiante. Or, la production de chaleur mesurée n'est que le résultat d'un certain nombre de processus physiologiques. Comme I'ont suggéré Close et Verstegen (1981) notamment, une meilleure connaissance de ces phénomènes internes et des réactions biochimiques mises en jeu permettrait de mieux interpréter les observations globales réalisées et ainsi de préciser les besoins nutritionnels des animaux. L'étude ci-dessous, réalisée chez le rat en croissance, concerne l'influence de la température ambiante sur la fixation d'énergie, sa répartition dans les protéines et les lipides, puis les variations dans les mêmes conditions de certains paramètres biochimiques du métabolisme.

\section{Matériels et méthodes.}

Au cours de 2 essais, 67 rats de souche Wistar CF sont utilisés une semaine après le sevrage, soit à un âge de 4 semaines environ et un poids moyen de $63 \pm 5 \mathrm{~g}$. Avant le début de l'expérience, les animaux sont maintenus à $21^{\circ} \mathrm{C}$. Ils sont ensuite soumis, sans transition, aux différentes températures expérimentales. Dans tous les cas, ils sont logés individuellement dans des cages métalliques, reçoivent de l'eau à volonté et bénéficient de $8 \mathrm{~h}$ de jour et $16 \mathrm{~h}$ de nuit. Les résultats obtenus sont traités par analyse de variance suivie d'un test $t$ pour la comparaison des movennes.

\section{Expérience 1.}

\section{- Animaux. Schéma expérimental.}

Dans un premier essai, 40 jeunes rats reçoivent à volonté pendant une semaine un régime préexpérimental (caséine $\mathrm{HCl}: 9,9$; $\mathrm{DL}$ méthionine : 0,08 ; 
amidon de maïs : 39,5 ; saccharose : 39,5 ; huile de maïs : 4,0 ; cellulose : 2,0 ; mélange minéral : 4,0; mélange vitaminique : 1,0) équilibré. Au terme de cette période, 28 rats d'un poids moyen de $85 \pm 5 \mathrm{~g}$ sont retenus pour l'expérimentation. Ils sont répartis selon la méthode des blocs complets en 4 lots homogènes d'après le poids et le gain de poids réalisés au cours de la période préexpérimentale.

Un premier lot de rats est sacrifié à l'issue de la mise en lots pour déterminer la composition corporelle initiale. Les animaux des 3 autres lots sont placés individuellement dans des cages à bilans maintenues respectivement à $3 \pm 1{ }^{\circ} \mathrm{C}$, $21 \pm 1^{\circ} \mathrm{C}$, et $31 \pm 1^{\circ} \mathrm{C}$; ils reçoivent alors, à volonté, pendant 18 jours, l'un des aliments dont la composition est présentée au tableau 1. Les rats placés à $31^{\circ} \mathrm{C}$ reçoivent un régime plus riche en protéines pour compenser la diminution spontanée des quantités d'aliment ingérées en milieu chaud. A la fin de l'essai, les animaux sont mis à jeun le soir à $17 \mathrm{~h}$ et sacrifiés le lendemain à $9 \mathrm{~h}$.

\section{TABLEAU 1}

Composition des régimes expérimentaux $(\mathrm{g} / \mathrm{kg})$.

\begin{tabular}{|c|c|c|}
\hline Température ambiante $\left({ }^{\circ} \mathrm{C}\right)$ & $3-21$ & 31 \\
\hline 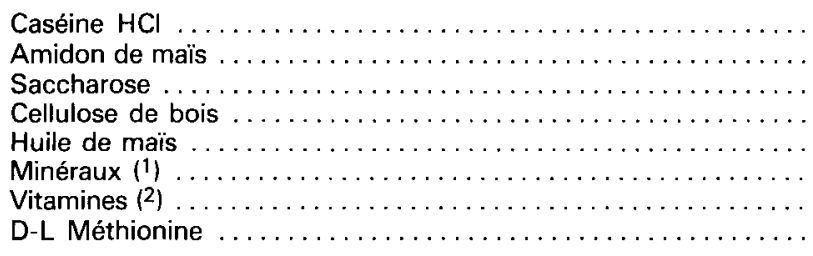 & $\begin{array}{l}154 \\
362,4 \\
362,4 \\
20 \\
50 \\
40 \\
10 \\
1,2\end{array}$ & $\begin{array}{l}250 \\
314,0 \\
314,0 \\
20 \\
50 \\
40 \\
10 \\
2,0\end{array}$ \\
\hline \multicolumn{3}{|l|}{ Résultats d'analyse : } \\
\hline 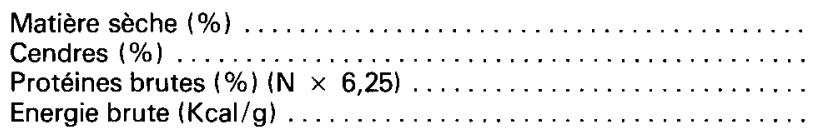 & $\begin{array}{r}95,37 \\
3,52 \\
15,43 \\
4,16\end{array}$ & $\begin{array}{r}95,55 \\
3,60 \\
23,71 \\
4,32\end{array}$ \\
\hline
\end{tabular}

(1) Composition $(\mathrm{g} / \mathrm{kg}$ ) du mélange minéral : Phosphate bicalcique : 373 ; Phosphate monopotassique : 198 ; Carbonate de calcium ; 263 ; Chlorure de sodium ; 88 ; Carbonate de magnésium ; 66 ; Sulfate ferreux $: 6,6$; Sulfate de cuivre $: 1,0 ;$ Sulfate de manganèse $; 2,7 ;$ Sulfate de zinc ; 1,$68 ;$ lodure de potassium : 0,01 ; Sulfate de cobalt : 0,01 .

(2) Composition $(\mathrm{g} / \mathrm{kg})$ du mélange vitaminique $\left({ }^{*}\right)$ : Vitamine B12:0,03; Acide folique : 0,10 ; Vitamine $\mathrm{K}: 0,50$; Thiamine : 0,80 ; Acide nicotinique : 3,00 ; Pyridoxine : 0,80 ; Riboflavine : 1,50; Inositol : 50,00; Acide paraaminobenzoïque : 50,00; Biotine : 0,02; Choline : 100,00 ; Vitamine E : 3,00 ; Acide pentothénique : 3,00 ; Amidon de maïs : 787,25.

$\left(^{*}\right)$ Les vitamines $A$ et $D$ sont administrées dans l'huile de maïs sous forme commerciale : $4 \mathrm{ml}$ de "Vitadone " Byla pour $1000 \mathrm{ml}$ d'huile.

\section{- Mesures et analyse.}

La quantité d'aliment ingérée est quotidiennement mesurée et le gain de poidus individuel déterminé par pesée bihebdomadaire. Tous les jours, les fécès 
sont récoltées, l'urine est recueillie dans l'acide sulfurique $\mathrm{N} / 10$ et conservée à $+4^{\circ} \mathrm{C}$ jusqu'à l'analyse.

L'énergie métabolisable est mesurée en retranchant de l'énergie brute ingérée la quantité d'énergie excrétée dans les fécès et dans l'urine. L'énergie excrétée par voie urinaire est estimée à partir de sa teneur en azote en utilisant l'équation de May et Nelson (1972) : $Y=7,00 X+6,56$, dans laquelle $Y$ représente l'énergie urinaire (Kcal) et $X$, I'azote total urinaire $(g)$. Les quantités d'énergie et d'azote fixées par l'animal sont mesurées par la méthode des analyses chimiques corporelles. Les contenus initiaux de la carcasse en différents constituants chimiques (azote et énergie notamment) sont déterminés par régression à partir du lot témoin, sacrifié au début de l'essai (Rérat et al., 1964). La production de chaleur des animaux est ensuite estimée par la différence entre l'énergie métabolisable et l'énergie retenue dans les tissus (Close, 1978). La quantité d'énergie retenue sous forme de protéines est calculée à partir du bilan azoté $15,7 \mathrm{Kcal} / \mathrm{g}$ de protéine déposée, selon Brouwer, 1965), tandis que l'énergie fixée sous forme de lipides est obtenue par différence entre l'énergie totale retenue et celle fixée sous forme de protéines.

Le dosage colorimétrique de l'urée dans l'urine est réalisé par la méthode au diacéthyl-monoxime en milieu fortement acide et en présence d'alun ferrique, à la longueur d'onde de $480 \mathrm{~nm}$ (Marsh et al., 1965). Les densités optiques enregistrées sont comparées à celle d'une gamme étalon.

\section{Expérience 2.}

\section{- Animaux. Schéma expérimental.}

27 rats sont répartis, selon leur poids vif, en 3 lots homogènes de même taille qui sont immédiatement placés dans les milieux thermiques expérimentaux (cf. expérience 1) pour une période de 3 semaines. L'aliment est fourni ad libitum. La composition des régimes est pratiquement la même que celle présentée au tableau 1, n'en différant que par le taux d'huile de maïs porté à $150 \mathrm{~g} / \mathrm{kg}$ et celui de l'amidon et du saccharose, réduit à $264 \mathrm{~g} / \mathrm{kg}$; d'où une teneur en énergie brute de $4,69 \mathrm{Kcal} / \mathrm{g}$ pour les régimes distribués à $3{ }^{\circ} \mathrm{C}$ et $21^{\circ} \mathrm{C}$ et de $4,84 \mathrm{Kcal} / \mathrm{g}$ à $31^{\circ} \mathrm{C}$.

\section{- Conditions de prélèvement et d'analyses.}

a) Prélèvement. - Au terme de la période expérimentale, les rats sont sacrifiés par décapitation sans jeûne préalable, entre $9 \mathrm{~h}$ et $11 \mathrm{~h}$. Le sang est recueilli sur héparine pour doser le glucose, le lactate, le glycérol et les acides gras libres plasmatiques. Les animaux sont ensuite rapidement disséqués et on prélève le foie, le cœur et le tissu adipeux épididymaire. Le foie est pesé, plongé dans l'azote liquide, puis conservé à $-20^{\circ} \mathrm{C}$, pour le dosage du glycogène et des lipides totaux. Le cour est débarrassé des oreillettes et des vaisseaux, puis congelé à $-20^{\circ} \mathrm{C}$. Les deux panicules adipeux épididymaires sont également prélevés et pesés. L'un d'entre eux sert à la détermination immédiate, à l'aide de glucose ${ }^{14} \mathrm{C}(\mathrm{U})$, des quantités de substrat transformé in vitro en $\mathrm{CO}_{2}$, acides gras et glycérol. L'activité de la lipoprotéine-lipase est mesurée sur le cœur et le panicule adipeux épididymaire ainsi congelés. 
b) Méthodes analytiques. - La teneur en protéines totales du plasma est déterminée par la méthode de Biuret (Gornall et al., 1949) en employant un étalon de sérum-albumine bovine. Le glucose plasmatique est dosé par la méthode à la glucose-oxydase, selon Huggett et Nixon (1957). Les taux d'acide lactique et de glycérol sont également mesurés par voie enzymatique en employant respectivement la lactate-déshydrogénase (LDH) et la mesure à $340 \mathrm{~nm}$ du NADH formé pour le premier, la glycérolkinase, la pyruvate-kinase et la LDH puis la mesure à $340 \mathrm{~nm}$ du NADH disparu pour le second. La teneur en acides gras libres du plasma est déterminée par titrimétrie suivant la méthode de Dole et Meinertz (1960) en utilisant le « bleu de Nil » comme indicateur coloré.

Dès le sacrifice des animaux, des coupes de tissu adipeux sont incubées pendant $2 \mathrm{~h}$ à $37{ }^{\circ} \mathrm{C}$, en présence de glucose uniformément marqué au ${ }^{14} \mathrm{C}$, selon la technique de O'Hea et Léveillé (1968) modifiée par Henry (non publié). On mesure ensuite la radioactivité du gaz carbonique produit (capté dans l'éthanolamine) et celle des acides gras et du glycérol synthétisés, après extraction des lipides selon la méthode de Folch et al. (1957).

Le glycogène hépatique est obtenu après digestion potassique à chaud, hydrolyse acide et dosage du glucose libéré par la méthode à la glucose-oxydase. La teneur en lipides du foie est obtenue par la technique d'extraction pondérale après décantation, à l'aide du mélange chloroforme-éthanol (Bligh et Dyer, 1959).

L'activité LPL est mesurée par une méthode voisine de celle décrite par de Gasquet et Pequignot (1972). Les fragments de tissus (100 à $300 \mathrm{mg}$ ) sont homogénéisés dans un tampon $\mathrm{NH}_{4} \mathrm{OH}-\mathrm{NH}_{4} \mathrm{Cl}$ hépariné $\mathrm{pH} 8,2$, puis I'homogénat est incubé pendant $1 \mathrm{~h}$ à $37^{\circ} \mathrm{C}$ en présence d'un substrat triglycéridique (intralipide à $10 \%$, laboratoire Vitrum, 94, Vitry, France) activé par du sérum de porc à raison de 1 volume pour 5 volumes de sérum. L'activité lipolytique est ensuite mesurée par le dosage des acides gras libérés suivant la méthode décrite ci-dessus.

TABLEAU 2

Influence du milieu thermique sur le niveau d'ingestion, la croissance pondérale et l'efficacité alimentaire.

Température ambiante $\left({ }^{\circ} \mathrm{C}\right)$

3

21

31

Signification statistique (1)

\begin{tabular}{|c|c|c|c|c|}
\hline $\begin{array}{l}\text { Ingérés : } \\
\text { - Matière sèche }(\mathrm{g} / \mathrm{j}) \ldots \ldots \ldots \\
\text { - Energie brute }(\mathrm{Kcal} / \mathrm{j}) \ldots \ldots \ldots \\
\text { - Protéines }(\mathrm{N} \times 6,25)(\mathrm{g} / \mathrm{j}) \ldots \ldots\end{array}$ & $\begin{array}{l}17,5 \mathrm{a} \pm 0,7(2) \\
76,4 \mathrm{a} \pm 3,0 \\
2,8 \mathrm{a} \pm 0,1\end{array}$ & $\begin{array}{r}14,5_{\mathrm{b}} \pm 0,2 \\
63,1_{\mathrm{b}} \pm 0,8 \\
2,3_{\mathrm{b}} \pm 0,0\end{array}$ & $\begin{array}{r}10,2 \mathrm{c} \pm 0,2 \\
46,3_{\mathrm{c}} \pm 1,0 \\
2,5_{\mathrm{c}} \pm 0,0\end{array}$ & $\begin{array}{c}* * \\
* * \\
*\end{array}$ \\
\hline Gain de poids $(\mathrm{g} / \mathrm{j}) \ldots \ldots \ldots \ldots$ & $3,3_{\mathrm{a}} \pm 0,2$ & $5,7_{\mathrm{b}} \pm 0,1$ & $5,4_{c} \pm 0,3$ & $* *$ \\
\hline $\begin{array}{l}\text { Efficacité alimentaire } \\
\text { (g de gain/g de MS ingérée) }(+) \text {. }\end{array}$ & $0,19_{\mathrm{a}} \pm 0,01$ & $0,40_{\mathrm{b}} \pm 0,00$ & $0,53_{c} \pm 0,02$ & $*^{*}$ \\
\hline
\end{tabular}

(1) Seuils de signification (NS, non significatif; ${ }^{*}, \mathrm{P}<0,05 ;{ }^{*}, \mathrm{P}<0,01$. a, b, c : les valeurs affectées d'une même lettre ne sont pas significativement différentes entre elles.

(2) Moyenne \pm écart-type de la moyenne $\left(\frac{s}{\sqrt{n}}\right.$, avec $s=$ écart-type, $n=$ effectif du lot $)$.

(+) MS : matière sèche : EB : énergie brute.

Reproduction Nutrition Développement $n^{\circ} 3-84$. - 11 . 


\section{Résultats.}

Expérience 1. - Par rapport aux performances obtenues à $21{ }^{\circ} \mathrm{C}$, l'exposition au froid s'accompagne d'une augmentation $(P<0,01)$ de $21 \%$ de la quantité de matière sèche ingérée, d'une diminution ( $P<0,01)$ de $42 \%$ de la vitesse de croissance et de $52 \%$ de l'efficacité alimentaire (tabl. 2). Au chaud, la consommation d'aliment sec est réduite $(P<0,01)$ de $30 \%$ et la vitesse de croissance de $5 \%(P<0,01)$, alors que l'efficacité alimentaire est améliorée ( $P<0,01)$ de $33 \%$ comparativement au milieu témoin.

La digestibilité apparente de l'azote alimentaire (tabl. 3) varie peu entre $3{ }^{\circ} \mathrm{C}$ et $21^{\circ} \mathrm{C}$, mais augmente de 2 points $(P<0,05)$ entre $21^{\circ} \mathrm{C}$ et $31^{\circ} \mathrm{C}$. Le coefficient de rétention azotée (CRN) est maximum à $21^{\circ} \mathrm{C}$ et diminue de façon significative $(P<0,01)$ à la fois à $3{ }^{\circ} \mathrm{C}(-49 \%)$ et à $31^{\circ} \mathrm{C}(-15 \%)$. En valeur absolue, les quantités journalières d'azote fixé sont comparables à $21^{\circ} \mathrm{C}$ et $31^{\circ} \mathrm{C}$ et plus faibles $(\mathrm{P}<0,05)$ à $3{ }^{\circ} \mathrm{C}$.

TABLEAU 3

Influence du milieu thermique sur la rétention azotée.

\begin{tabular}{|c|c|c|c|c|}
\hline Température ambiante $\left({ }^{\circ} \mathrm{C}\right)$ & 3 & 21 & 31 & $\begin{array}{l}\text { Signification } \\
\text { statistique }\left({ }^{1}\right)\end{array}$ \\
\hline $\begin{array}{l}\mathrm{N} \text { ingéré }(\mathrm{mg} / \mathrm{j}) \ldots \ldots \ldots \ldots \\
\text { CUDN }(\%)(3) \ldots \ldots \ldots \\
\mathrm{N} \text { total urinaire }(\mathrm{mg} / \mathrm{j}) \ldots \ldots \ldots \\
\mathrm{N} \text { urée urinaire }(\mathrm{mg} / \mathrm{j}) \ldots \ldots \ldots \\
\mathrm{N} \text { retenu }(\mathrm{mg} / \mathrm{j}) \ldots \ldots \ldots \ldots \ldots \ldots \\
\mathrm{CRN}(\%)(4) \ldots \ldots \ldots \ldots \ldots\end{array}$ & $\begin{array}{l}454 \mathrm{a} \pm 17(2) \\
91,2 \mathrm{a} \pm 0,6 \\
183_{\mathrm{a}} \pm 11 \\
164 \mathrm{a} \pm 10 \\
127_{\mathrm{a}} \pm 10 \\
30,5 \mathrm{a} \pm 1,6\end{array}$ & $\begin{aligned} & 374_{\mathrm{b}} \pm 14 \\
& 91,8_{\mathrm{a}} \pm 0,5 \\
& 72_{\mathrm{b}} \pm 3 \\
& 49_{\mathrm{b}} \pm 3 \\
& 206_{\mathrm{b}} \pm 5 \\
& 59,9 \pm 0,8\end{aligned}$ & $\begin{array}{l}406_{\mathrm{b}} \pm 9 \\
93,9_{\mathrm{b}} \pm 0,3 \\
154_{\mathrm{a}} \pm 7 \\
117_{\mathrm{c}} \pm 4 \\
195_{\mathrm{b}} \pm 10 \\
51,0 \pm 1,8\end{array}$ & $\begin{array}{l}* \\
* \\
* * \\
* \\
* \\
* \\
*\end{array}$ \\
\hline
\end{tabular}

(1) et (2) : voir tableau 2.

(3) CUDN : coefficient d'utilisation digestive apparente de l'azote.

(3) CRN : coefficient de rétention azotée.

L'élimination globale d'azote par voie urinaire est doublée $(P<0,01)$ à $3{ }^{\circ} \mathrm{C}$ et à $31^{\circ} \mathrm{C}$ par rapport à une température ambiante de $21^{\circ} \mathrm{C}$. La quantité d'azote uréique est également accrue $(P<0,01)$ à $3{ }^{\circ} \mathrm{C}$ et à $31{ }^{\circ} \mathrm{C}$ par rapport au milieu témoin, tout en étant plus élevée $(P<0,01)$ au froid qu'au chaud.

La figure 1 montre notamment que la quantité d'énergie retenue augmente $(P<0,01)$ avec l'élévation de la température ambiante, à l'inverse de l'énergie métabolisable ingérée $(P<0,01)$. La quantité d'énergie fixée sous forme de protéines est du même ordre à $21^{\circ} \mathrm{C}$ et à $31^{\circ} \mathrm{C}$ et significativement inférieure $(P<0,01)$ à $3^{\circ} \mathrm{C}$. Par contre, l'énergie fixée sous forme de lipides augmente $(P<0,01)$ régulièrement avec la température ambiante entre $3^{\circ} \mathrm{C}$ et $31^{\circ} \mathrm{C}$.

La composition chimique corporelle des animaux est présentée au tableau 4. La teneur en protéines semble indépendante du milieu thermique. Le taux de lipi- 
des des carcasses est comparable à $3{ }^{\circ} \mathrm{C}$ et à $21^{\circ} \mathrm{C}$ et significativement inférieur $(\mathrm{P}<0,01)$ à celui des rats placés à $31^{\circ} \mathrm{C}$.

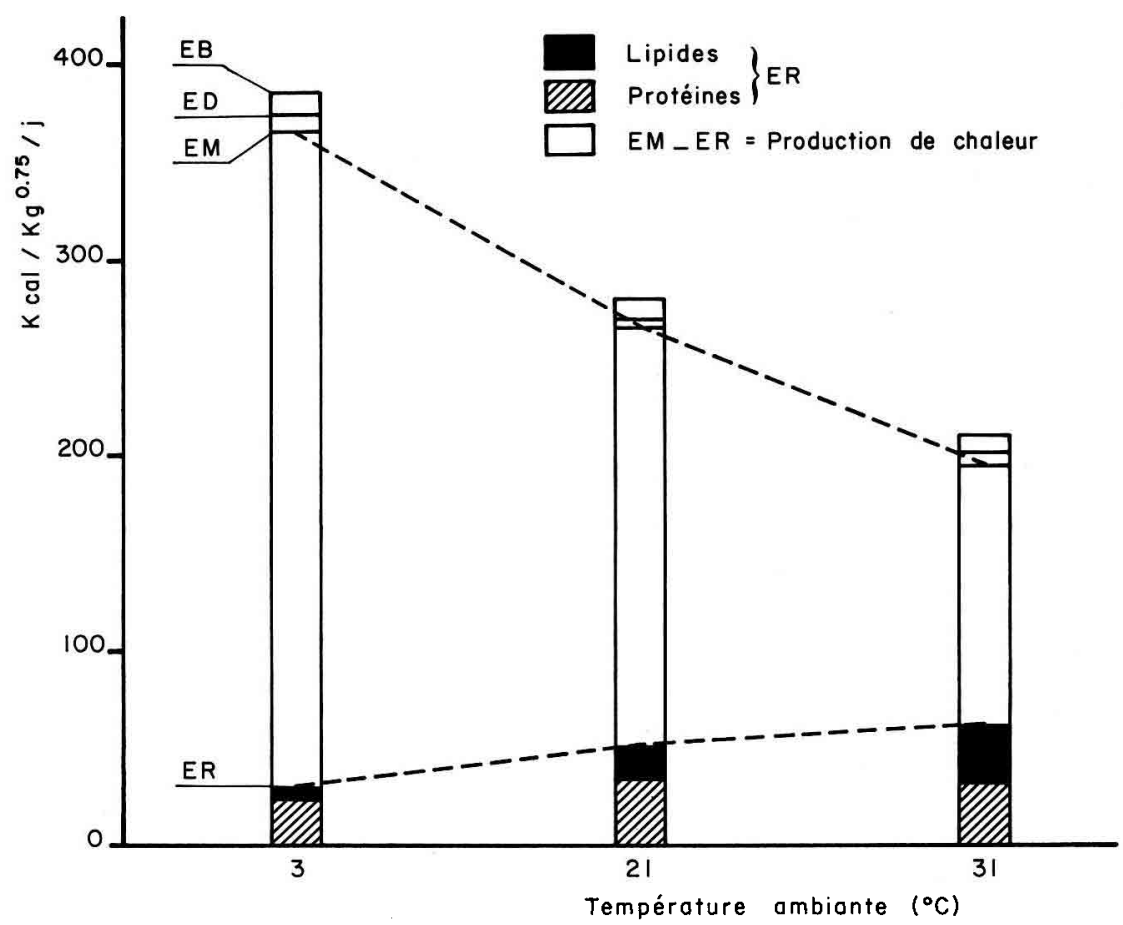

FIG. 1. - Température ambiante et utilisation de l'énergie chez le rat en croissance.

$E B$ : énergie brute, ED : énergie digestible ; EM : énergie métabolisable ; ER : énergie retenue.

TABLEAU 4

Influence du milieu thermique sur la composition corporelle du rat en croissance.

Température ambiante $\left({ }^{\circ} \mathrm{C}\right)$

3

21

31

Signification statistique ( $\left.{ }^{1}\right)$

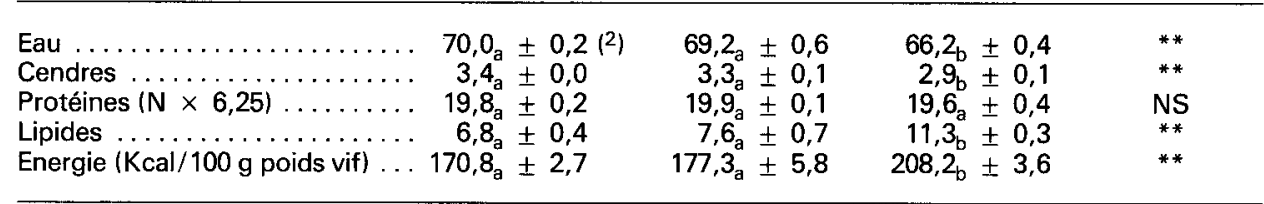

(1) et $(2)$ : voir tableau 2.

Expérience 2. - Les données fournies par cet essai en matière de performances de croissance et de consommation sont comparables à celles de l'expérience précédente et sont présentées dans une publication antérieure (Christon, 1979). Seuls sont considérés ici les résultats relatifs aux paramètres biochimiques. 
Aucune variation notable du taux de protéines sériques ni de la glycémie n'est observée en fonction de la température d'élevage (tabl. 5). La teneur en glycogène du foie est significativement abaissée $(P<0,05)$ sous l'effet du froid et reste inchangée à température élevée, alors que sa teneur en lipides diminue aussi bien à $3{ }^{\circ} \mathrm{C}$ qu'à $31^{\circ} \mathrm{C}$. La concentration plasmatique d'acide lactique est augmentée $(P<0,05)$ à $3{ }^{\circ} \mathrm{C}$ et peu affectée par une température ambiante de $31^{\circ} \mathrm{C}$, par rapport au milieu témoin. La glycérolémie est sensiblement accrue ( $P<0,05$ ) au froid et un peu moins au chaud, tandis que la teneur en acides gras libres plasmatiques $n^{\prime}$ est pratiquement pas modifiée à $3^{\circ} \mathrm{C}$, comparativement à $21^{\circ} \mathrm{C}$, mais augmente de façon significative $(P<0,05)$ à $31^{\circ} \mathrm{C}$.

\section{TABLEAU 5}

Variations de quelques paramètres sanguins et des réserves hépatiques en fonction de la température ambiante.

\begin{tabular}{|c|c|c|c|}
\hline Température ambiante $\left({ }^{\circ} \mathrm{C}\right)$ & 21 & 31 & $\begin{array}{l}\text { Signification } \\
\text { statistique }\left({ }^{1}\right)\end{array}$ \\
\hline 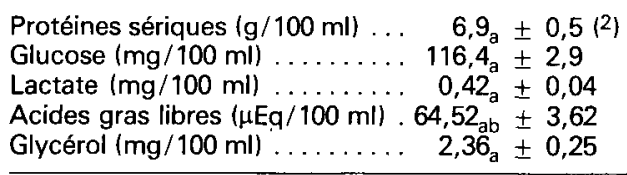 & $\begin{aligned} 8,2 & \pm 0,4 \\
118,0 & \pm 2,9 \\
0,29 & \pm 0,04 \\
59,71_{a} & \pm 3,05 \\
1,35_{b} & \pm 0,21\end{aligned}$ & $\begin{aligned} 8,3 & \pm 0,9 \\
118,0_{a} & \pm 3,9 \\
0,31_{b} & \pm 0,01 \\
79,00 b & \pm 6,72 \\
2,14_{a b} & \pm 0,13\end{aligned}$ & $\begin{array}{l}\text { NS } \\
\text { NS } \\
* \\
* \\
*\end{array}$ \\
\hline $\begin{array}{l}\text { Glycogène hépatique } \\
\quad \mathrm{mg} / \mathrm{g} \text { de foie } \ldots \ldots \ldots \ldots \ldots \ldots, 22,9,9_{\mathrm{a}} \pm 3,6 \\
\text { total }(\mathrm{mg}) \ldots \ldots \ldots \ldots \ldots \ldots, 129,3_{\mathrm{a}} \pm 22,7\end{array}$ & $\begin{array}{c}35,6_{a} \pm 4,7 \\
342,1_{b} \pm 46,2\end{array}$ & $\begin{array}{c}38,3_{b} \pm 3,0 \\
305,5_{b} \pm 25,2\end{array}$ & $\stackrel{*}{*}$ \\
\hline $\begin{array}{l}\text { Lipides totaux du foie } \\
\quad \mathrm{mg} / \mathrm{g} \text { de foie } \ldots \ldots \ldots \ldots \ldots \ldots, 41,5_{\mathrm{a}} \pm 4,7 \\
\quad \text { total (mg) . . . } \ldots \ldots \ldots \ldots, 229,0_{\mathrm{a}} \pm 26,9\end{array}$ & $\begin{array}{c}49,6_{b} \pm 3,4 \\
434,0_{b} \pm 36,6\end{array}$ & $\begin{aligned} 37,4 a & \pm 0,2 \\
295,3_{a} & \pm 16,4\end{aligned}$ & $\stackrel{*}{*}$ \\
\hline
\end{tabular}

(1) et (2) : voir tableau 2.

Chez les rats témoins, l'activité de la lipoprotéine-lipase (fig. 2) est beaucoup plus élevée $(P<0,05)$ dans le myocarde qu'au niveau du tissu adipeux. II en est de même à la température ambiante de $3^{\circ} \mathrm{C}$ mais pas à $31^{\circ} \mathrm{C}$. Dans le muscle cardiaque, l'activité $L P L$ augmente $(P<0,01)$ de manière importante sous l'influence $d^{\prime} u n$ milieu froid et, au contraire, diminue $(P<0,01)$ sous l'effet du chaud. Dans le tissu adipeux, l'activité LPL augmente aussi bien à $3^{\circ} \mathrm{C}$ $(\mathrm{P}<0,01)$ qu'à $31^{\circ} \mathrm{C}(\mathrm{P}<0,10)$.

L'examen de la figure 3 montre qu'après deux heures d'incubation, plus de $50 \%$ du glucose ${ }^{14} \mathrm{C}(\mathrm{U})$ incorporé dans le tissu adipeux épididymaire est transformé en acides gras et en glycérol quel que soit le milieu thermique. La capacité du tissu gras de réserve à oxyder le glucose (production de $\mathrm{CO}^{2}$ ), ou à l'incorporer dans les constituants lipidiques, varie peu entre 21 et $31^{\circ} \mathrm{C}$; elle est, par contre, trois ou quatre frois plus grande pour les tissus des animaux élevés dans un milieu froid $\left(3^{\circ} \mathrm{C}\right)$. 


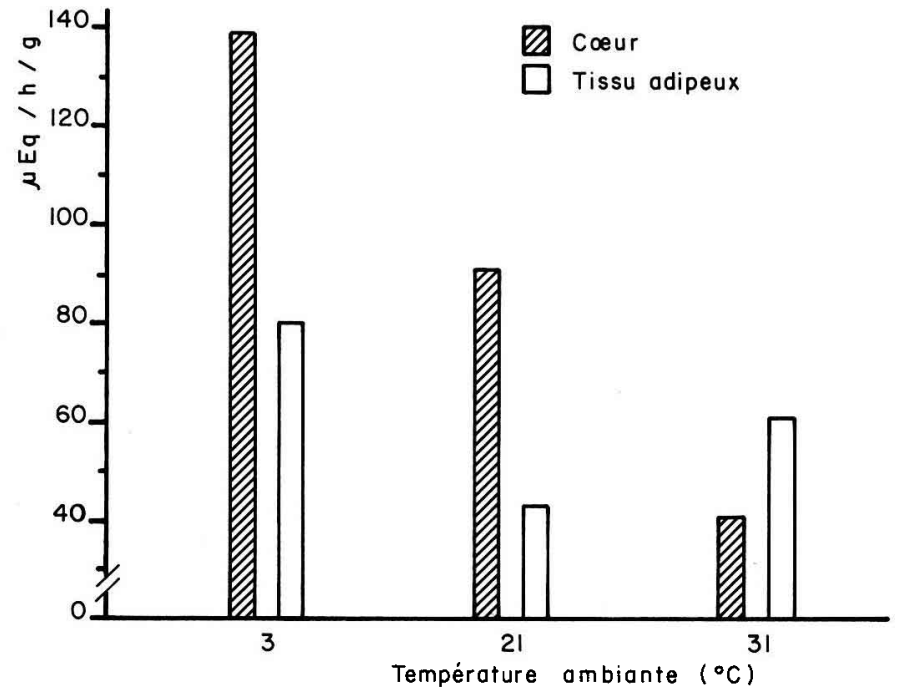

FIG. 2. - Effet du milieu thermique sur l'activité spécifique (acides gras libérés/g de tissu frais) de la lipoprotéine lipase du cceur et du tissu adipeux blanc.

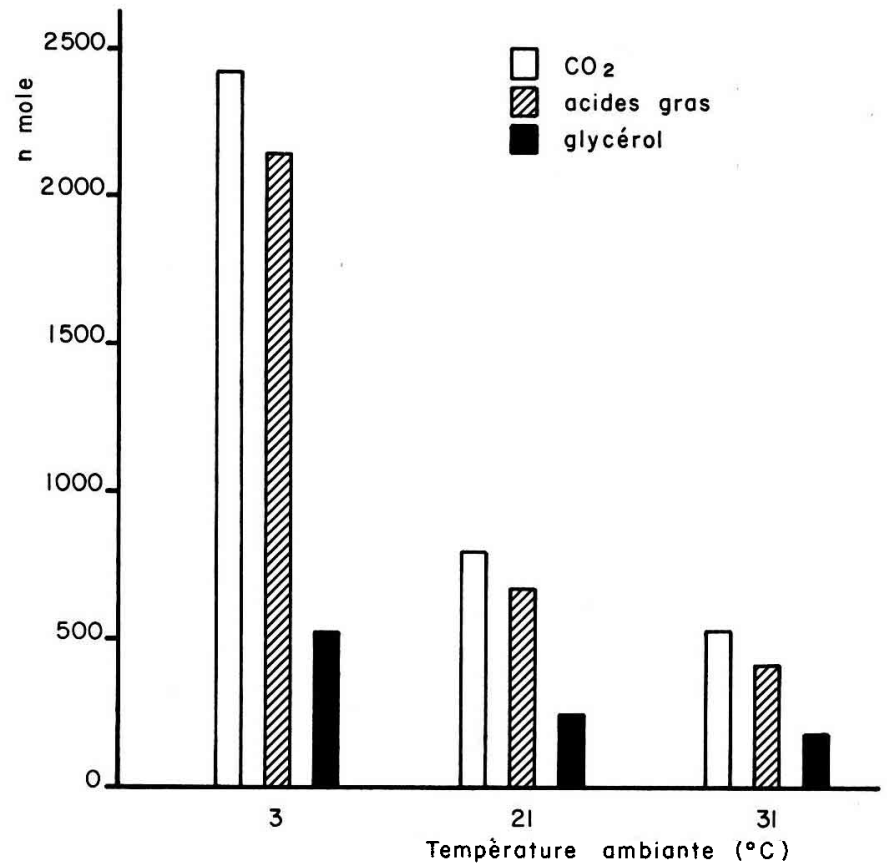

FIG. 3. - Influence de la température ambiante sur la transformation in vitro de g/ucose ${ }^{14} \mathrm{C}(\mathrm{u})$, en $\mathrm{CO}_{2}$ acides gras et glycérol au niveau du tissu adipeux blanc. 


\section{Discussion.}

Nos résultats montrent que la quantité d'aliment ingérée par le rat en croissance (à un poids vif moyen de $120 \mathrm{~g}$ ) est significativement accrue au froid et, au contraire, diminuée au chaud, confirmant notamment ceux de Harris (1982) et de Jakubczak (1976). II s'ensuit une variation comparable des quantités d'énergie métabolisable ingérées (EMi). Toutefois, à la température ambiante de $3^{\circ} \mathrm{C}$, seuls $8 \%$ de l'énergie métabolisable sont fixés dans les tissus contre $19 \%$ à $21{ }^{\circ} \mathrm{C}$ (en accord avec Kotowski, 1973). Les animaux placés en ambiance froide utilisent donc, par rapport aux témoins, une proportion plus importante de l’EMi à des fins de production de chaleur pour maintenir leur homéothermie. Inversement, à $31^{\circ} \mathrm{C}$, en accord avec Alhassan et al. (1976), la quantité d'énergie retenue s'élève à $31 \%$ de l'EMi, en liaison vraisemblablemen avec la diminution du métabolisme basal (Chaffee et Roberts, 1971) et une activité physique réduite (Pennycuik, 1964 ; Hartsook et Nee, 1976) généralement observées en milieu chaud.

Par ailleurs, à $3{ }^{\circ} \mathrm{C}$, la rétention azotée est diminuée d'environ $50 \%$ par rapport au milieu témoin. Cette observation, qui correspond à celles faites notamment par Young et Cook (1955) chez le Rat et par Fuller et Boyne (1971) chez le porc, est corroborée par une excrétion urinaire d'azote accrue, principalement sous forme d'urée, traduisant une accélération du catabolisme protéique en milieu froid (Beaton, 1963 ; Whitten et al., 1970 ; Smith, 1976). Ce phénomène, lié à l'augmentation de la thermogenèse, conduit à une réduction de la quantité d'énergie disponible pour les synthèses et il en découle une diminution marquée de l'efficacité alimentaire et de la croissance pondérale aux basses températures (Sellers et al., 1954 ; Comberg et al., 1972). D'autre part, bien que la quantité des protéines ingérées ne soit pas tout à fait la même dans les différents milieux thermiques, la rétention azotée, rapportée à l'azote absorbé, est également inférìeure en ambiance chaude à celle des rats témoins. Toutefois, la différence est bien moindre, loin d'atteindre, par exemple, celle qui est observée par Holmes (1973, 1974) chez le porc et nos résultats ne montrent qu'une faible diminution (non significative) de la quantité en valeur absolue d'azote fixé. L'augmentation de l'excrétion urinaire d'azote à $31^{\circ} \mathrm{C}$ correspond donc plutôt à une dégradation de protéines excédentaires (Christon, 1979) traduisant une réduction de la synthèse protéique, probablement sous contrôle hormonal. II s'ensuit, malgré l'accroissement de l'efficacité alimentaire et de la quantité d'énergie retenue en milieu chaud, une diminution du gain de poids, liée en fait à la teneur en eau des tissus déposés. Précisément, en accord avec Senault-Bournique et al. (1967), nous observons un dépôt lipidique maximum à $31^{\circ} \mathrm{C}$ et d'autant plus faible que le milieu est plus froid. Cependant, aux basses températures, la teneur en lipides des carcasses n'est pas significativement différente de celle observée dans le milieu de référence, tout comme chez le porc nourri à volonté (Verstegen et al., 1982).

Les résultats obtenus à la suite de l'exploration du métabolisme lipidique permettent d'expliquer en partie les phénomènes observés dans nos conditions expérimentales. Ainsi, l'exposition au froid s'accompagne d'une augmentation de la lipolyse due à la stimulation de la lipase tissulaire déclenchée par une sécrétion 
accrue de catécholamines (Hsieh et Carlson, 1957 ; Leduc, 1961). Or, nous observons notamment une élévation de la glycérolémie qui, en accord avec Therriault et al. (1969), témoigne d'une mobilisation des réserves lipidiques chez le rat placé à $3{ }^{\circ} \mathrm{C}$. Chez ces mêmes animaux, le taux d'acides gras libres plasmatiques n'est pas significativement supérieur à celui des témoins, en raison de leur rapide captation par les tissus (Hannon et Larson, 1962 ; Beauvallet, Portet et Solier, 1976). Cette captation est confirmée par l'augmentation, au froid, de l'activité LPL tant au niveau des organes utilisateurs d'acides gras comme le cœur ou les muscles squelettiques (Begin-Heick et Heick, 1977) que du tissu adipeux de réserve (en accord avec Bertin et al., 1977). Nous observons, de plus, une incorporation accrue du glucose dans les acides gras du tissu adipeux blanc (étude in vitro), montrant ainsi l'intensification des phénomènes de synthèse et de dépôt lipidiques chez le jeune rat adapté au froid. Cela s'accorde parfaitement avec l'augmentation des activités enzymatiques liées à la production de NADPH (Goubern et Portet, 1981) et l'accélération du turn-over des triglycérides dans le tissu adipeux de réserve montrée par Portet et al. (1975) chez le rat élevé au froid. La diminution de la teneur en lipides totaux hépatiques, également observée par Treadwell et al. (1957) et Portet et al. (1967) dans des conditions comparables, est précisément la conséquence d'une utilisation active des acides gras pour la thermogenèse.

A l'inverse, en milieu chaud, le taux plasmatique d'acides gras libres est significativement augmenté chez le rat comme chez le porc (Hsia, 1974) ; ce fait est à rapprocher de la réduction, montrée par Cassuto et Chaffee (1966) chez le Hamster placé à température élevée, des activités enzymatiques impliquées dans l'oxydation des acides gras. Ce résultat correspondrait, par conséquent, à une utilisation réduite des lipides en milieu chaud. D'autres observations conduisent aussi à le penser, notamment la diminution montrée ici de l'activité LPL dans le myocarde et, au contraire, sa stimulation dans le tissu adipeux. Ce dernier fait est également retrouvé chez le porcelet placé à une température ambiante supérieure de $4^{\circ} \mathrm{C}$ à sa température de thermoneutralité (CKriston, données non publiées). Enfin, la baisse du taux de lipides circulańts (Jouanneteau et Peres, 1975) de même que l'élévation du quotient respiratoire (Hluszko et al., 1969) chez le rat maintenu dans un milieu chaud témoignent également d'une dégradation réduite des matières grasses aux températures élevées. II s'ensuit un accroissement du dépôt gras également observé chez d'autres espèces, notamment chez le porc (Holmes, 1971). En outre, l'incorporation de glucose marqué dans le tissu adipeux n'étant pas stimulée sous l'effet de la chaleur, on peut penser que l'augmentation du dépôt lipidique chez le rat blanc placé dans ces conditions thermiques est principalement liée à une synthèse hépatique suivie d'un transfert en direction du tissu adipeux de réserve. La diminution de la teneur en lipides totaux hépatiques constatée chez les animaux placés à $31{ }^{\circ} \mathrm{C}$ n'est pas en contradiction avec cette hypothèse. Mais d'autres mesures seraient nécessaires pour l'étayer et pour mieux expliquer le développement accru du tissu adipeux en ambiance chaude.

A côté de ces changements majeurs, les autres variations constatées au niveau des paramètres biochimiques étudiés montrent, dans chaque milieu thermique, une remarquable adaptation des réactions de l'animal en vue de maintenir son homéostasie. En effet, en accord avec les observations de Beaton (1963), la 
teneur en protéines plasmatiques reste invariable quelle que soit la température ambiante, en dépit du catabolisme protéique aux températures extrêmes. De même, grâce en particulier à une mobilisation active du glycogène hépatique à $3{ }^{\circ} \mathrm{C}$, la glycémie demeure inchangée dans les trois milieux thermiques envisagés. Cependant, cela n'est pas sans poser quelques problèmes en milieu froid, comme en témoigne la teneur élevée en acide lactique plasmatique observée chez les rats placés à $3{ }^{\circ} \mathrm{C}$ et chez bien d'autres espèces dans des conditions similaires (Tulea et al., 1977). En fait, aux basses températures, l'acide lactique provenant de la dégradation du glycogène musculaire sous I'action des catécholamines (Dordea et al., 1977) est essentiellement utilisé pendant le frisson thermique qui suit immédiatement l'exposition au froid (Minaire et Chatonnet, 1966); en dehors de cette phase, il s'accumule dans le plasma.

En conclusion, les résultats obtenus montrent l'importance du rôle joué par le climat dans l'utilisation métabolique de l'énergie et de l'azote alimentaires. De plus, l'effet propre du milieu thermique nous a paru déterminant sur des paramètres zootechniques fondamentaux comme la croissance, l'efficacité alimentaire et la composition corporelle. Ainsi, pour augmenter sa production de chaleur, I'animal placé en ambiance froide présente une accélération générale du métabolisme, associé à un catabolisme azoté accru. De ce fait, peu d'énergie reste disponible pour la synthèse protéique et le développement du tissu musculaire se trouve limité, si bien que, malgré la surconsommation observée aux basses températures, la croissance est fortement ralentie. En milieu chaud, en dépit d'une réduction marquée du niveau d'ingestion, on observe un accroissement notable du tissu adipeux, associé à une sous-utilisation des acides gras. En outre, la diminution de la vitesse de croissance, de même que l'augmentation des quantités d'azote urinaire chez les animaux placés à température élevée sont loin d'être expliquées. Finalement, ces quelques observations posent avec acuité, d'une part, le problème de la régulation hormonale de l'adaptation aux milieux chauds, d'autre part, celui de l'ajustement quantitatif, mais aussi probablement qualitatif, des besoins nutritionnels en fonction de l'ambiance thermique de l'animalerie.

Reçu en octobre 1983. Accepté en janvier 1984.

\section{Références}

ALHASSAN W. S., BUCHANAN-SMITH J. G., ASHTON G. C., 1976. Relation of high temperature regimes to energy metabolism, body composition and feed and water intake in rats. J. anim. Sci., 42, 608-615.

BEATON J. P., 1963. Metabolic effects of dietary protein level with caloric restriction in coldexposed rats. Can. J. Biochem. Physiol., 41, 149-160.

BEAUVALLET M., PORTET R., SOLIER M., 1976. Variations des triglycérides et des acides gras libres plasmatiques chez le rat, en fonction de la durée de maintien au froid. C. R. Soc. Biol., 170, 300-303.

BEGIN-HEICK N., HEICK H. M. C., 1977. Increased lipoprotein-lipase activity of skeletal muscle in cold-acclimated rats. Can. J. Biochem., 55, 1241-1243.

BERTIN R., GOUBERN M., PORTET R., 1977. Effects of diets and cold acclimation on lipoproteinlipase activity and cyclic nucleotide levels in some tissues of rats. In GIRARDIER L., SEYDOUX I., Effectors of thermogenesis, Experientia, Suppl. 32, 185-189. 
BLIGH E., DYER W. J., 1959. A rapid method of total lipid extraction and purification. Can. J. Biochem. Physiol., 37, 911-917.

BROUWER E., 1965. Report of subcommittee on constants and factors. In BLAXTER K. L., Energy metabolism, Acad. Press, New York and London.

CASSUTO Y., CHAFFEE R. R. J., 1966. Effects of prolonged heat exposure on the hamster. Am. J. Physiol., 210, 423-426.

CHAFFEE R. R., ROBERTS J. C., 1971. Temperature acclimation in birds and mammals. Ann. Rev. Physiol., 33, 155-202.

CHRISTON R., 1979. Influence de la température ambiante sur le métabolisme énergétique et azoté du rat en croissance. Th. Doct. $3^{\mathrm{e}}$ cycle $(64 \mathrm{pp}$.). Univ. de Paris Vt.

CLOSE W. H., 1978. The effects of plane of nutrition and environmental temperature on energy metabolism of the growing pig. 3. The efficiency of energy utilization for maintenance and growth. Br. J. Nutr., 40, 433-438.

CLOSE W. H., VERSTEGEN M. W. A., 1981. Factors influencing thermal losses in nonruminants : a review. Livest. Prod. Sci, 8, 449-463.

COMBERG G., PLISCHKE R., WEGNER W., FEDER H., 1972. Die auswirkunde Temperaturen $\left(+8{ }^{\circ} \mathrm{C}\right)$ auf die Leistungen von Pietrains, Belgischer Landrasse und deren Kreuzungen.

1. Mitteilung: Mastleistungseigenschaften und Shlachtköperbewertung. Zuchtungskunde, 44, 91-97.

DOLE V. P., MEINERTZ H., 1960. Microdetermination of long chain fatty acids in plasma and tissues. J. biol. Chem., 235, 2595-2599.

DORDEA M., PORA E. A., PERSECA T., POP M., 1977. Changes in carbohydrate and fat metabolism in the hamster (Cricetus cricetus) acclimated to cold. Rev. roum. Biol.-Biol. anim., 22, 161-163.

FOLCH J., LEES M., SLOANE-STANDLEY G. H., 1957. A simple method for the isolation and purification of total lipids from animals tissus. J. biol. chem., 226, 497-509.

FULLER M. F., BOYNE A. W., 1971. The effects of environmental temperature on the growth and metabolism of pigs given different amounts of food. 1. Nitrogen metabolism, growth and body composition. Br. J. Nutr., 25, 259-272.

GASQUET P. de, PEOUIGNOT E., 1972. Lipoprotein-lipase activities in adipose tissues, heart and diaphragm of the genetically obese mouse (ob/ob). Biochem. J., 127, 445-447.

GORNALL A. G., BARDAWILL C. J., DAVID M. M., 1949. Determination of serum proteins by means of the biuret reaction. J. biol. Chem., 177, 751-766.

GOUBERN M., PORTET R., 1981. Modulation of malic enzyme and glucose-6 phosphate deshydrogenase activities in some tissues of cold acclimated rats. Comp. biochem. Physiol. (B), 69, 237-241.

HANNON J. P., LARSON A. M., 1962. Fatty acid metabolism during norepinephrine-induced thermogenesis in the cold-acclimatized rat. Am. J. Physiol., 203, 1055-1061.

HARRIS R. B. S., 1982. The food intake of Zuker rats in relation to environmental temperature. J. Physiol., 324, 58-59.

HARTSOOK E. N., NEE J. C. M., 1976. Effects in the rat of environmental temperature, diet dilution and treadmill running on voluntary food intake, body composition and endocrine organ mass : a multiple regression analysis. J. Nutr., 106, 1314-1325.

HILLER H. H., GEORGIEV Sh., JOBST E., 1977. Die Nährstoff und Eneriebedarffsdeckung von Ratten bei unterschiedlichen Ungebungstemperaturen. Z. Versuchstierk, 19, 79-84.

HLUSZKO M. T., PORTET R., CHEVILLARD L., 1969. Bilan énergétique chez les rats adaptés à $5{ }^{\circ} \mathrm{C}$ ou $30^{\circ} \mathrm{C}$ et soumis à des régimes contenant $5 \%$ ou $30 \%$ de lipides. C. R. Soc. Biol., 163, 344-348.

HOLMES C. W., 1971. Growth and backfat depth of pigs kept at high temperature. Anim. Prod., 13, 521-527.

HOLMES C. W., 1973. The energy and protein metabolism of pigs growing at a high ambiant temperature. Anim. Prod., 16, 117-133.

HOLMES C. W., 1974. Further studies on the energy and protein metabolism of pigs growing at a high ambiant temperature, including measurements with fasting pigs. Anim. Prod., 19, 211220.

HOLMES C. W., CLOSE W. H., 1977. The influence of climatic variables on energy metabolism 
and associated aspects of productivity in the pig, 51-73. In HARESIGN W., SWAN H., LEWIS D., Nutrition and the climatic environment. Butterworths, London.

HUGGETT A. S. G., NIXON D. A., 1957. Enzymatic determination of blood glucose. Biochem. J., 66, $12 \mathrm{p}$.

HSIA L. C., 1974. A climatisation of pigs to high temperature. Thes. Sci. Univ. Aberdeen.

HSIEH A. C. L., CARLSON L. D., 1957. Role of adrenaline and noradrenaline in chemical regulation of heat production. Am. J. Physiol., 190, 243-246.

JAKUBCZAK L. F., 1976. Fooed and water intakes of Rats as a function of strain, age temperature and body weight. Physiol. Behav., 17, 251-258.

JENSEN A. H., BECKER D. E., COX J. L., GEHLBACH G. D., HERMON B. G., 1963. Some management and environmental factors affecting swine performance. J. anim. Sci, 22, 1111 (Abstr.).

JOUANNETEAU J., PERES G., 1975. Particularités du métabolisme lipidique du rat soumis à une augmentation de la température ambiante. J. Physiol. Paris, 71, 336A.

KOTOWSKI J., 1973. Badania bilansowe nad wplywem temperatury na straty roznych form energii u szczurow albinosow. Zeszyty Naukowe, Akademi Rolniczej w Szczecinie, 41, 269286.

LEDUC J., 1961. Catecholamine production and release in exposure and acclimation to cold. Acta physiol. scand., 53 (Suppl. 183).

MARSH W. H., FINGERHUT B., MILLER H., 1965. Automated and manual direct methods for the determination of blood urea. Clin. Chem., 11, 624.

MAY M. A., NELSON T. S., 1972. Correlation of nitrogen to the heat of combustion of rat urine. J. anim. Sci, 35, 38-40.

MINAIRE Y., CHATONNET J., 1966. L'effet glycogénolytique peut-il à lui seul expliquer l'action calorigénique très intense que l'adrénaline exerce lors du frisson thermique maximum ? Arch. Sci. physiol., 20, 21-41.

O'HEA E. K., LÉVEILLÉ G. A., 1968. Lipogenesis in isolated adipose tissue of the domestic chick (Gallus domesticus). Comp. Biochem. Physiol., 26, 1081-1089.

PENNYCUIK P. R., 1964. The effects on rats of chronic exposure to $34^{\circ} \mathrm{C}$. Part 1. Aust. J. biol. Sci., 17, 208-219.

PORTET R., SENAULT-BOURNIQUE C., CHEVILLARD L., 1967. Teneur en lipides et en cholestérol du foie du Rat adapté à différentes températures et soumis à divers régimes alimentaires. C. R. Soc. Biol., 161, 268-272.

PORTET R., HLUSZKO M. T., SENAULT C., 1975. Nutritional aspects of lipid metabolism in brown adipose tissue of cold-acclimated rats. Proc. Xth. int. Congr. Nutr., Kyoto, 193-194.

RÉRAT A., FÉVRIER C., HENRY Y., LOUGNON J., 1964. Evolution de la composition corporelle chez le rat blanc en croissance. Ann. Biol. anim. Bioch. Biophys., 4, 35-47.

SELLERS E. A., YOU R. W., MOFFAT N. M., 1954. Regulation of food consumption by caloric value of the ration in rats exposed to cold. Am. J. Physiol., 17, 367-371.

SENAULT-BOURNIQUE C., PORTET R., CHEVILLARD L., 1967. Lipides totaux de la carcasse chez le rat adapté à différentes températures et soumis à divers régimes alimentaires. $C$. $R$. Soc. Biol. Paris, 161, 233.

SMITH O. K., 1976. Rise in plasma -amino nitrogen concentration in rats eviscerated after cold exposure. Am. J. Physiol., 231, 174-178.

THERRIAULT D. G., HUBBARD R. W., MELLIN D. B., 1969. Endocrine control of fat mobilization in the isolated fat cells of cold-exposed rats. Lipids, 4, 413-420.

TREADWELL C. R., FLICK D. F., VAHOUNY G. V., 1957. Nutrition studies in the cold. 1. Influence of diet and low environmental temperature on growth and on the lipid content of livers in the rat. J. Nutr., 63, 611-622.

TULEA E., SCHNEIDER Fr., PETROIU A., SCHNEIDER F., 1977. Investigations concerning the metabolic adaptation of rats to cold. Rev. roum. Morphol. Embryol. Physiol., 14, 113-114.

VERSTEGEN M. W. A., ClOSE W. H., START J. B., MOUNT L. E., 1973. The effects of environmental temperature and plane of nutrition on heat loss, energy retention and deposition of protein and fat in groups of growing pigs. Br. J. Nutr., 30, 21-35.

VERSTEGEN M. W. A., BRASCAMP E. W., VAN DER HEL W., 1978. Fattening of pigs in relation to climatic environment, housing and feeding level. Can. J. anim. Sci., 58, 1-13. 
VERSTEGEN M. W. A., BRANDSMA H. A., METEMAN G., 1982. Feed requierment of growing pigs at low environmental temperatures. J. anim. Sci, 55, 88-94.

WEBSTER A. J. F., 1976. Effects of cold on energy metabolism of sheep, 218-226. In H. D. JOHNSON H. D., Progress in animal biometeorology, Vol. 1, Part. 1. Swets and Zeitlinger, Amsterdam.

WEBSTER A. J. F., GORDON J. G., SMITH J. S., 1976. Energy exchanges of veal calves in relation to body weight, food intake and air temperature. Anim. Prod., 23, 35-42.

WHITTEN B. K., BURLINGTON R. F., POSIVIATA M. A., SIDEL C. M., BEECHER G. R., 1970. Amino acid catabolism in environmental extremes effect of temperature and calories. Am. J. Physiol., 219, 1046-1049.

YOUNG D. R., COOK S. F., 1955. Effect of environmental temperature and dietary protein on urinary nitrogen excretion of rats. Proc. Soc. exp. Biol. Med., 89, 482-484. 